\title{
THE ASYMMETRIC PRODUCT OF THREE INHOMOGENEOUS LINEAR FORMS
}

\author{
A. C. WoODS
}

(Received 4 September 1979, revised 18 December 1980)

Communicated by A. J. van der Poorten

\begin{abstract}
It is shown, given any positive real number $\lambda$ and any point $\left(x_{1}, x_{2}, x_{3}\right)$ of $R_{3}$ and any lattice $\Lambda$ in $\boldsymbol{R}_{3}$; that there exists a point $\left(z_{1}, z_{2}, z_{3}\right)$ of $\Lambda$ for which

$$
-d(\Lambda) / 8 \lambda<\left(z_{1}+x_{1}\right)\left(z_{2}+x_{2}\right)\left|z_{3}+x_{3}\right|<\lambda d(\Lambda) / 8,
$$

which generalizes a theorem due to Remak.

1980 Mathematics subject classification (Amer. Math. Soc.): 10 E 15.

Let $\Lambda$ denote a lattice in euclidean 3-space $R_{3}$, so that $\Lambda$ is the free group generated by three linearly independent points $X_{1}, X_{2}, X_{3}$ of $R_{3}$. The number $\left|\operatorname{det}\left(X_{1}, X_{2}, X_{3}\right)\right|$ is called the determinant $d(\Lambda)$ of $\Lambda$, and is independent of the choice of basis. Let $I I X$ denote the product of the coordinates of a point $X$ in $R_{3}$. A theorem of Remark [12] asserts that, given any point $X \in R_{3}$, there exists a point $Z \in \Lambda$ such that

$$
|\Pi(Z+X)| \leqslant d(\Lambda) / 8
$$

the equality sign being necessary if and only if $X \equiv \frac{1}{2}\left(z_{1}, \ldots, z_{n}\right) \bmod \Lambda$, where $\left(z_{1}, 0, \ldots, 0\right),\left(0, z_{2}, 0, \ldots, 0\right), \ldots,\left(0, \ldots, 0, z_{n}\right)$ is a basis of $\Lambda$.

Simplifications of Remak's proof have been given by Davenport [5] and Mahler [8], while different proofs have been given by Birch and SwinnertonDyer [1] and Narzullaev [11]. Our objective is to give a proof of the following extension of Remak's theorem.
\end{abstract}

OCopyright Australian Mathematical Society 1981 
THEOREM 1. Given any lattice $\Lambda$ and any point $X=\left(x_{1}, x_{2}, x_{3}\right)$ in $R_{3}$, to any positive real number $\lambda$ there corresponds a point $Z=\left(z_{1}, z_{2}, z_{3}\right)$ of $\Lambda$, depending on $\lambda$, such that

$$
-d(\Lambda) / 8 \lambda^{2}<\left(z_{1}+x_{1}\right)\left(z_{2}+x_{2}\right)\left|z_{3}+x_{3}\right|<\lambda^{2} d(\Lambda) / 8 .
$$

The equality sign is necessary if and only if either

(i) $\lambda=1$ and $X \equiv \frac{1}{2}\left(z_{1}, z_{2}, z_{3}\right) \bmod \Lambda$, where $\left(z_{1}, 0,0\right),\left(0, z_{2}, 0\right),\left(0,0, z_{3}\right)$ form a basis of $\Lambda$; or

(ii) $\lambda^{4}=(m+1) /(m-1)$ or $(m-1) /(m+1)$ where $m=2,3, \ldots$ and for some positive numbers $t_{1}, t_{2}, t_{3}, \Lambda$ has a basis of the form

$$
\left(t_{1}\left(\lambda+\lambda^{-1}\right), t_{2}\left(\lambda-\lambda^{-1}\right), 0\right),\left(t_{1}\left(\lambda-\lambda^{-1}\right), t_{2}\left(\lambda+\lambda^{-1}\right), 0\right),\left(0,0, t_{3}\right)
$$

and $X \equiv\left(-t_{1} \lambda,-t_{2} \lambda, \frac{1}{2} t_{3}\right) \bmod \Lambda$.

The method of proof is the projective one due to Birch and Swinnerton-Dyer [1]. Define the homogeneous minimum of $\Lambda$ as $m_{h}(\Lambda)=\inf |\Pi Z|$ extended over all points $Z$ of $\Lambda$ other than the origin, where $\Pi Z$ denotes the product of the coordinates of $Z$. By the projective method, Theorem 1 follows if

(a) it is true when $m_{h}(\Lambda)=0$, and

(b) it is true when $m_{h}(\Lambda)$ is a positive attained minimum.

Proof OF (a).

LEMMA 1. If $\Lambda$ is a lattice in $R_{2}$ and $\lambda>0$, then given any point $X \in R_{2}$, there exists a point $Z$ of $\Lambda$ such that

$$
-d(\Lambda) / 4 \lambda^{2}<\Pi(X+Z)<\lambda^{2} d(\Lambda) / 4 .
$$

The equality sign is necessary if and only if either

(i) $\lambda=1$ and $X \equiv \frac{1}{2}\left(z_{1}, z_{2}\right) \bmod \Lambda$, where $\left(z_{1}, 0\right),\left(0, z_{2}\right)$ form a basis of $\Lambda$; or

(ii) $\lambda^{4}=(m+1) /(m-1)$ or $(m-1) /(m+1)$ where $m=2,3, \ldots$ and for some positive numbers $t_{1}, t_{2}, \Lambda$ has a basis of the form $\left(t_{1}\left(\lambda+\lambda^{-1}\right), t_{2}\left(\lambda-\lambda^{-1}\right)\right)$, $\left(t_{1}\left(\lambda-\lambda^{-1}\right), t_{2}\left(\lambda+\lambda^{-1}\right)\right)$. Further, if $\Lambda$ has a basis of this form than necessarily $X \equiv-\left(t_{1} \lambda, t_{2} \lambda\right) \bmod \Lambda$.

The case $\lambda=1$ is due to Minkowski [10], and the first part for general $\lambda$ is due to Davenport [6]. The enumeration of the best possible cases is due to Blaney [2].

LEMMA 2. If $\Lambda$ is a lattice in $R_{2}$ and $\lambda>0$, then given any point $X=\left(x_{1}, x_{2}\right) \in$ $R_{2}$, there exists a point $Z=\left(z_{1}, z_{2}\right)$ of $\Lambda$ such that

$$
-d(\Lambda) / 4 \lambda^{2}<\left(z_{1}+x_{1}\right)\left|z_{2}+x_{2}\right|<\lambda^{2} d(\Lambda) / 4 \text {. }
$$


The equality sign is necessary if and only if $\lambda=1$ and $X$ and $\Lambda$ are as given in Lemma 1.

The case $\lambda=1$ is again Minkowski's theorem while the extension to general $\lambda$ is contained in [14].

By a standard transformation we mean a linear map of the type $x_{1} \rightarrow t_{1} x_{1}$, $x_{2} \rightarrow t_{2} x_{2}, \ldots, x_{n} \rightarrow t_{n} x_{n}$ where $t_{1}, t_{2}, \ldots, t_{n}$ are positive real numbers such that $t_{1} t_{2} \cdots t_{n}=1$.

LeMMA 3. Let $\Lambda$ be a lattice in $R_{n}$ for which $m_{h}(\Lambda)=0$. After a permutation of the coordinates there exists an integer $p$ with $1<p<n-1$ and a sequence of standard transformations $T_{k}$ such that the sequence of lattices $T_{k}(\Lambda)$ converges in the sense of Mahler [9], to a lattice $\Lambda_{0}$ with a basis of the form $X_{i}=\left(x_{i j}\right)$, $i, j=1,2, \ldots, n$, where $x_{i j}=0$ for $1<i<p$ and $p+1<j<n$.

This lemma is due to Birch and Swinnerton-Dyer [1].

LEMMA 4. If $a_{1}, a_{2}, a_{3} ; b_{1}, b_{2}, b_{3} ; m$ are positive real numbers such that $a_{1} a_{2} a_{3} \geqslant m$ and $b_{1} b_{2} b_{3} \geqslant m$ then

$$
\prod_{i=1}^{2}\left(a_{i} b_{3}+b_{i} a_{3}\right) \geqslant 2 m\left(a_{3}+b_{3}\right)
$$

with equality if and only if $a_{i}=b_{i}$ for $i=1,2,3$ and $a_{1} a_{2} a_{3}=m$.

This is proved in [14].

By a divided cell in $R_{n}$ we mean a generalized paallelopiped having a vertex in the interior of each generalized coordinate octant. Such a divided cell is said to be regular if any line segment joining two vertices of the cell and crossing exactly one coordinate plane is necessarily an edge of the cell.

Lemma 5. If $C$ is a divided cell in $R_{2}$ of area $A(C)$ then at least one of its vertices $\left(x_{1}, x_{2}\right)$ satisfies

$$
-A(C) / 4 \lambda^{2}<x_{1} x_{2} \leqslant \lambda^{2} A(C) / 4
$$

with strict inequality unless the vertices of $C$ are of the form $\pm\left(t_{1} \lambda, t_{2} \lambda\right)$, $\pm\left(t_{1} \lambda^{-1}, t_{2} \lambda^{-1}\right)$ where $t_{1}, t_{2}$ are positive numbers such that $t_{1} t_{2}=\frac{1}{4} A(C)$.

The proof of this is due to Cassels [3] based on a method due to Sawyer [13]. 
LEMMA 6. If $C$ is a regular divided cell in $R_{3}$ of volume $V(C)$ then at least one of its vertices $\left(x_{1}, x_{2}, x_{3}\right)$ satisfies

$$
-V(C) / 8 \lambda^{2}<x_{1} x_{2}\left|x_{3}\right|<\lambda^{2} V(C) / 8,
$$

with strict inequality unless the vertices of $C$ are of the form

$$
\begin{gathered}
\pm\left(t_{1} \lambda, t_{2} \lambda, t_{3}\right), \quad \pm\left(t_{1} \lambda^{-1}, t_{2} \lambda^{-1},-t_{3}\right), \\
\pm\left(t_{1} \lambda^{-1},-t_{2} \lambda^{-1}, t_{3}\right) \text { and } \pm\left(t_{1} \lambda, t_{2} \lambda,-t_{3}\right),
\end{gathered}
$$

where $t_{1}, t_{2}, t_{3}$ are positive numbers such that $t_{1} t_{2} t_{3}=V(C) / 8$.

Proof. Assume that all the vertices of $C$ lie outside the region consisting of the points $\left(x_{1}, x_{2}, x_{3}\right)$ such that

$$
-V(C) / 8 \lambda^{2}<x_{1} x_{2}\left|x_{3}\right|<\lambda^{2} V(C) / 8
$$

Let $P=\left(y_{1}, y_{2}, y_{3}\right)$ and $Q=\left(z_{1}, z_{2},-z_{3}\right)$ be two vertices of $C$ such that $y_{1} z_{1}>$ $0, y_{2} z_{2}>0, y_{3}>0$ and $z_{3}>0$. Project $C$ into the coordinate plane $x_{3}=0$, parallel to the edge $P Q$ of the cell $C$. This projection yields a divided cell $D$ of $R_{2}$ with area $A(D)=V(C)\left(y_{3}+z_{3}\right)^{-1}$. The vertex $(x, y, 0)$ of $D$ obtained from the projection of $P$ is given by

$$
x=\left(y_{3}+z_{3}\right)^{-1}\left(y_{1} z_{3}+y_{3} z_{1}\right),
$$

If $y_{1} y_{2}>0$, then by the initial assumption,

$$
y_{1} y_{2} y_{3}>\lambda^{2} V(C) / 8 \quad \text { and } \quad z_{1} z_{2} z_{3}>\lambda^{2} V(C) / 8
$$

and therefore also, by Lemma 4

$$
x y>\left(y_{3}+z_{3}\right)^{-1} \lambda^{2} V(C) / 4 .
$$

However, if $y_{1} y_{2}<0$, the initial assumption implies that

$$
y_{1} y_{2} y_{3} \leqslant-V(C) / 8 \lambda^{2} \quad \text { and } \quad z_{1} z_{2} z_{3} \leqslant-V(C) / 8 \lambda^{2},
$$

and, again by Lemma 4 ,

$$
x y \leqslant-\left(y_{3}+z_{3}\right)^{-1} V(C) / 4 \lambda^{2},
$$

contrary to Lemma 5 . This proves Lemma 6, except when the equality sign is needed; in which case, Lemma 4 implies that $y_{i}=z_{i}$ for $i=1,2,3$; and $\left|y_{1} y_{2} y_{3}\right|=\lambda^{2} V(C) / 8$. The projection of $P$ is then $\left(z_{1}, z_{2}, 0\right)$. Lemma 5 then implies that $\left(z_{1}, z_{2}\right)$ is one of the points $\pm\left(t_{1} \lambda, t_{2} \lambda\right), \pm\left(t_{1} \lambda^{-1}, t_{2} \lambda^{-1}\right)$, where $t_{1} t_{2}=V(C) / 8 z_{3}$, which completes the proof of Lemma 6 .

Let $\Lambda$ be a lattice in $R_{3}$ and let $X \in R_{3}$. We say $\Lambda+X$ has a divided cell if eight points of $\Lambda+X$ form a regular divided cell of volume $d(\Lambda)$. 
Lemma 7. If $\Lambda+X$ has a regular divided cell, then Theorem 1 is true for these values of $\Lambda$ and $X$.

Proof. Let $C$ denote a regular divided cell of $\Lambda+X$. By Lemma 6, Theorem 1 is true with strict inequality unless the vertices of $C$ are of the form $\pm\left(t_{1} \lambda, t_{2} \lambda, t_{3}\right), \pm\left(-t_{1} \lambda^{-1}, t_{2} \lambda^{-1}, t_{3}\right), \pm\left(t_{1} \lambda^{-1},-t_{2} \lambda^{-1}, t_{3}\right)$ and $\pm\left(-t_{1} \lambda,-t_{2} \lambda, t_{3}\right)$, where $t_{1}, t_{2}, t_{3}$ are positive numbers such that $t_{1} t_{2} t_{3}=d(\Lambda) / 8$. Then $\Lambda$ contains the two-dimensional sublattice $\Lambda^{\prime}$ generated by

$$
\left(t_{1}\left(\lambda+\lambda^{-1}\right), t_{2}\left(\lambda-\lambda^{-1}\right), 0\right), \quad\left(t_{1}\left(\lambda-\lambda^{-1}\right), t_{2}\left(\lambda+\lambda^{-1}\right), 0\right),
$$

with $d\left(\Lambda^{\prime}\right)=d(\Lambda) / 2 t_{3}$. Therefore, by Lemma 1 , with $X$ taken as $\left(-t_{1} \lambda,-t_{2} \lambda\right)$, there exists a point $Z^{\prime}=\left(z_{1}^{\prime}, z_{2}^{\prime}\right)$ of $\Lambda^{\prime}$, such that

$$
-d(\Lambda) / 8 \lambda^{2}<\left(z_{1}^{\prime}-t_{1} \lambda\right)\left(z_{2}^{\prime}-t_{2} \lambda\right)\left|t_{3}\right| \leqslant \lambda^{2} d(\Lambda) / 8 \text {. }
$$

As $\left(z_{1}^{\prime}-t_{1} \lambda, z_{2}^{\prime}-t_{2} \lambda, t_{3}\right) \in \Lambda+X$, Theorem 1 is true with strict inequality, unless the equality sign holds, in which case Lemma 1 implies that $\lambda$ is one of the listed exceptional values, and hence $\Lambda+X$ is one of the exceptional cases listed in Theorem 1.

THEOREM 1A. If $\Lambda$ is a lattice in $R_{3}$ with $m_{h}(\Lambda)=0$, then Theorem is true for $\Lambda$.

Proof. By Lemma 3, there exists, after a permutation of coordinates, an integer $p=1$ or 2 , and a sequence of standard transformations $T_{k}$, such that the sequence of lattices $T_{k}(\Lambda)$ converges, in the sense of Mahler [9], to a lattice $\Lambda_{0}$ with a basis of the form $Z_{i}=\left(x_{i 1}, x_{i 2}, x_{i 3}\right), i=1,2,3$, where $x_{i j}=0$ for $1 \leqslant i \leqslant$ $p$ and $p+1 \leqslant j \leqslant 3$. Denote by $\Lambda_{0}^{(1)}$ the $p$-dimensional lattice of basis $\left(x_{i 1}, x_{i 2}, \ldots, x_{i p}\right), i=1,2, \ldots, p$. Further, denote by $\Lambda_{0}^{(2)}$ the $3-p$ dimensional lattice of basis $\left(x_{i, p+1}, \ldots, x_{i 3}\right), i=p+1, \ldots, 3$.

The point $X$ of Theorem 1 may be replaced by any point congruent to $X$ modulo $\Lambda$. Also, the truth of Theorem 1 for $\Lambda$ and $X$ is equivalent to the truth of Theorem 1 for $T_{k}(\Lambda)$ and $T_{k}(X)$ after some permutation of the coordinates independent of $k$. As $T_{k}(\Lambda) \rightarrow \Lambda_{0}$ as $k \rightarrow \infty$, it follows that $T_{k}(X)$ may be chosen from a bounded region independent of $k$. Therefore, replacing the sequence $\left\{T_{k}\right\}$ by a subsequence, if necessary, we may assume that $T_{k}(X)$ converges to a point $\bar{X}=\left(\bar{x}_{1}, \bar{x}_{2}, \bar{x}_{3}\right)$, say.

As Theorem 1 remains unchanged when the $x_{1}$ and $x_{2}$ coordinates are interchanged, we may, without loss of generality, assume that the permutation of coordinates, given by Lemma 3, is one of the three $\left(x_{1}, x_{2}, x_{3}\right) \rightarrow\left(x_{1}, x_{2}, x_{3}\right)$ or $\left(x_{1}, x_{3}, x_{2}\right)$ or $\left(x_{3}, x_{2}, x_{1}\right)$. As $p$ may be 1 or 2 , this results in six cases, which we consider individually in what follows. 
Case 1. $\left(x_{1}, x_{2}, x_{3}\right) \rightarrow\left(x_{1}, x_{2}, x_{3}\right)$ and $p=1$. By Lemma 1, there exists a point $\left(u_{2}, u_{3}\right) \in \Lambda_{0}^{(2)}$, such that

$$
u=\left|\left(u_{2}+\bar{x}_{2}\right)\left(u_{3}+\bar{x}_{3}\right)\right|<\frac{1}{4} d\left(\Lambda_{0}^{(2)}\right),
$$

the equality sign being necessary only if $\Lambda_{0}^{(2)}$ has a basis of the form $\left(z_{2}, 0\right)$, $\left(0, z_{3}\right)$, where $\left(\bar{x}_{2}, \bar{x}_{3}\right) \equiv \frac{1}{2}\left(z_{2}, z_{3}\right)$ modulo $\Lambda_{0}^{(2)}$.

Let $m, n$ be integers for which

$$
\left(u_{2}, u_{3}\right)=m\left(x_{22}, x_{23}\right)+n\left(x_{32}, x_{33}\right),
$$

and put $x_{1}^{*}=m x_{21}+n x_{31}+\bar{x}_{1}$.

If $u=0$, then, for any $u_{1} \in \Lambda_{0}^{(1)}$,

$$
\left(u_{1}+x_{1}^{*}\right)\left(u_{2}+\bar{x}_{2}\right)\left|u_{3}+\bar{x}_{3}\right|=0 \text {, }
$$

and as $\left(u_{1}+m x_{21}+n x_{31}, u_{2}, u_{3}\right) \in \Lambda_{0}$, Theorem 1 holds for $\Lambda$ and $X$. Thus, we may assume that $u \neq 0$.

Suppose, first, that $u_{2}+\bar{x}_{2}>0$.

As $d\left(\Lambda_{0}^{(1)}\right) d\left(\Lambda_{0}^{(2)}\right)=d\left(\Lambda_{0}\right)$, so

$$
\frac{1}{2} d\left(\Lambda_{0}^{(1)}\right) \leqslant \frac{d\left(\Lambda_{0}\right)}{8\left(u_{2}+\bar{x}_{2}\right)\left|u_{3}+\bar{x}_{3}\right|} .
$$

There exists $u_{1} \in \Lambda_{0}^{(1)}$, such that

$$
-d\left(\Lambda_{0}^{(1)}\right) / 2 \lambda^{2} \leqslant\left(u_{1}+x_{1}^{*}\right)<\lambda^{2} d\left(\Lambda_{0}^{(1)}\right) / 2,
$$

with strict inequality unless $\lambda=1$ and $x_{1}^{*}=\frac{1}{2} d\left(\Lambda_{0}^{(1)}\right)$ modulo $d\left(\Lambda_{0}^{(1)}\right)$. Therefore

$$
-d\left(\Lambda_{0}\right) / 8 \lambda^{2} \leqslant\left(u_{1}+x_{1}^{*}\right)\left(u_{2}+\bar{x}_{2}\right)\left|u_{3}+\bar{x}_{3}\right|<\lambda^{2} d\left(\Lambda_{0}\right) / 8 \text {. }
$$

If the inequality is strict, then, for sufficiently large $k, T_{k}(\Lambda+X)$ satisfies Theorem 1 , and therefore also, $\Lambda+X$ satisfies Theorem 1 , with strict inequality. Otherwise, $\lambda=1$, and $\Lambda_{0}^{(2)}$ has a basis of the form $\left(z_{2}, 0\right),\left(0, z_{3}\right), z_{2}>0, z_{3}>0$, and $\left(\bar{x}_{2}, \bar{x}_{3}\right) \equiv \frac{1}{2}\left(z_{2}, z_{3}\right)$ modulo $\Lambda_{0}^{(2)}$. We may assume that $\bar{X}$ is chosen modulo $\Lambda_{0}$, so that $\left(\bar{x}_{2}, \bar{x}_{3}\right)=-\frac{1}{2}\left(z_{2}, z_{3}\right)$, and then we could take $u_{2}=0$ or $z_{2}, u_{3}=0$ or $z_{3}$, with the corresponding $x_{1}^{*} \equiv \frac{1}{2} d\left(\Lambda_{0}^{(1)}\right)$ modulo $d\left(\Lambda_{0}^{(1)}\right)$.

Hence $\Lambda_{0}+\bar{X}$ has a regular divided cell with vertices $\frac{1}{2}\left( \pm d\left(\Lambda_{\delta}^{(1)}\right), \pm z_{2}, \pm z_{3}\right)$. By convergence, $T_{k}(\Lambda+X)$ has a regular divided cell for all sufficiently large $k$, and so, by Lemma 7 , the theorem follows.

Therefore, we may assume that $u_{2}+\bar{x}_{2}<0$.

There exists $u_{1} \in \Lambda_{0}^{(1)}$ such that

$$
-\frac{1}{2} \lambda^{2} d\left(\Lambda_{0}^{(1)}\right)<u_{1}+x_{1}^{*}<\frac{1}{2} \lambda^{-2} d\left(\Lambda_{0}^{(1)}\right),
$$

with strict inequality unless $\lambda=1$ and $x_{1}^{*} \equiv \frac{1}{2} d\left(\Lambda_{0}^{(1)}\right)$ modulo $\Lambda_{0}^{(1)}$. As

$$
\frac{1}{2} d\left(\Lambda_{0}^{(1)}\right) \leqslant-\frac{d\left(\Lambda_{0}\right)}{8\left(u_{2}+\bar{x}_{2}\right)\left|u_{3}+\bar{x}_{3}\right|},
$$


we again obtain

$$
-\frac{d\left(\Lambda_{0}\right)}{8 \lambda^{2}}<\left(u_{1}+x_{1}^{*}\right)\left(u_{2}+\bar{x}_{2}\right)\left|u_{3}+x_{3}\right| \leqslant \frac{\lambda^{2} d\left(\Lambda_{0}\right)}{8} .
$$

The rest of the argument parallels that given for $u_{2}+\vec{x}_{2}>0$.

Case 2. $\left(x_{1}, x_{2}, x_{3}\right) \rightarrow\left(x_{1}, x_{2}, x_{3}\right)$ and $p=2$. Since $\Lambda_{0}^{(2)}$ is one-dimensional, there exists an integer $n$ such that

$$
-\frac{1}{2} d\left(\Lambda_{0}^{(2)}\right)<n x_{33}+\bar{x}_{3} \leqslant \frac{1}{2} d\left(\Lambda_{0}^{(2)}\right) .
$$

Put $x_{1}^{*}=n x_{31}+\bar{x}_{1}$ and $x_{2}^{*}=n x_{32}+\bar{x}_{2}$. By Lemma 1, there exists a point $\left(u_{1}, u_{2}\right) \in \Lambda_{0}^{(1)}$ such that

$$
-\frac{d\left(\Lambda_{0}^{(1)}\right)}{4 \lambda^{2}}<\left(u_{1}+x_{1}^{*}\right)\left(u_{2}+x_{2}^{*}\right)<\frac{\lambda^{2} d\left(\Lambda_{0}^{(1)}\right)}{4},
$$

and therefore also

$$
-\frac{d(\Lambda)}{8 \lambda^{2}}<\left(u_{1}+x_{1}^{*}\right)\left(u_{2}+x_{2}^{*}\right)\left|n x_{33}+\bar{x}_{3}\right| \leqslant \frac{\lambda^{2} d(\Lambda)}{8} .
$$

As $\left(u_{1}+n x_{31}, u_{2}+n x_{32}, n x_{33}\right) \in \Lambda$, if the inequality is strict, then Theorem 1A follows as before. Otherwise, by Lemma $1, \Lambda_{0}^{(1)}$ has a basis of the form $\left(t_{1}\left(\lambda+\lambda^{-1}\right), t_{2}\left(\lambda-\lambda^{-1}\right)\right),\left(t_{1}\left(\lambda-\lambda^{-1}\right), t_{2}\left(\lambda+\lambda^{-1}\right)\right)$, for some positive real numbers $t_{1}, t_{2}$. Moreover, $\left(x_{1}^{*}, x_{2}^{*}\right) \equiv\left(-t_{1} \lambda,-t_{2} \lambda\right)$ modulo $\Lambda_{0}^{(1)}$, and further, $n x_{33}+\bar{x}_{3}$ $=\frac{1}{2} d\left(\Lambda_{0}^{(2)}\right)$. Then $\Lambda_{0}+\bar{X}$ contains the points

$$
\pm\left(t_{1} \lambda^{-1},-t_{2} \lambda^{-1}, \frac{1}{2} d\left(\Lambda_{\delta}^{(2)}\right)\right), \pm\left(t_{1} \lambda, t_{2} \lambda, \frac{1}{2} d\left(\Lambda_{0}^{(2)}\right)\right) \text {. }
$$

Now $(n-1) x_{33}+\bar{x}_{3}=-\frac{1}{2} d\left(\Lambda_{0}^{(2)}\right)$, so put $x_{1}^{\prime}=(n-1) x_{31}+\bar{x}_{1}, \quad x_{2}^{\prime}=$ $(n-1) x_{32}+\bar{x}_{2}$. Applying Lemma 1 , there exists a point $\left(u_{1}^{\prime}, u_{2}^{\prime}\right) \in \Lambda_{0}^{(1)}$, such that

$$
-\frac{d\left(\Lambda_{\delta}^{(1)}\right)}{4 \lambda^{2}}<\left(u_{1}^{\prime}+x_{1}^{\prime}\right)\left(u_{2}^{\prime}+x_{2}^{\prime}\right) \leqslant \frac{\lambda^{2} d\left(\Lambda_{\delta}^{(1)}\right)}{4},
$$

and therefore also,

$$
-\frac{d(\Lambda)}{8 \lambda^{2}}<\left(u_{1}^{\prime}+x_{1}^{\prime}\right)\left(u_{2}^{\prime}+x_{2}^{\prime}\right)\left|(n-1) x_{33}+\bar{x}_{3}\right|<\frac{\lambda^{2} d(\Lambda)}{8} .
$$

If the inequality is strict, Theorem $1 \mathrm{~A}$ follows. Otherwise, since we already know a basis for $\Lambda_{0}^{(1)}$, Lemma 1 implies that

$$
\left(x_{1}^{\prime}, x_{2}^{\prime}\right) \equiv\left(-t_{1} \lambda,-t_{2} \lambda\right) \text { modulo } \Lambda_{0}^{(1)} \text {, }
$$

and therefore also, that $\Lambda_{0}+\bar{X}$ contains the points

$$
\pm\left(t_{1} \lambda^{-1},-t_{2} \lambda^{-1},-\frac{1}{2} d\left(\Lambda_{0}^{(2)}\right)\right), \quad \pm\left(t_{1} \lambda, t_{2} \lambda,-\frac{1}{2} d\left(\Lambda_{0}^{(1)}\right)\right) \text {. }
$$


These four points, together with the four points (i), comprise the vertices of a regular divided cell of $\Lambda_{0}+\bar{X}$. By Lemma 7, Theorem $1 \mathrm{~A}$ follows as before.

Case 3. $\left(x_{1}, x_{2}, x_{3}\right) \rightarrow\left(x_{1}, x_{3}, x_{2}\right)$ and $p=1$. By Lemma 1, there exists a point $\left(u_{2}, u_{3}\right) \in \Lambda_{0}^{(2)}$ such that

$$
\left|\left(u_{2}+\bar{x}_{2}\right)\left(u_{3}+\bar{x}_{3}\right)\right| \leqslant \frac{1}{4} d\left(\Lambda_{\delta}^{(2)}\right) .
$$

The proof now parallels that of Case 1, dividing into cases according to the sign of $u_{3}+\bar{x}_{3}$.

Case 4. $\left(x_{1}, x_{2}, x_{3}\right) \rightarrow\left(x_{1}, x_{3}, x_{2}\right)$ and $p=2$. As in Case 2, there exists an integer $n$ such that

$$
-\frac{1}{2} d\left(\Lambda_{\delta}^{(2)}\right)<n x_{33}+\bar{x}_{3} \leqslant \frac{1}{2} d\left(\Lambda_{\delta}^{(2)}\right) \text {. }
$$

Put $x_{1}^{*}=n x_{31}+\bar{x}_{1}, x_{2}^{*}=n x_{32}+\bar{x}_{2}$, and suppose first that $n x_{33}+\bar{x}_{3}>0$. By Lemma 2, there exists $\left(u_{1}, u_{2}\right) \in \Lambda_{0}^{(1)}$ such that

$$
-\frac{d\left(\Lambda_{0}^{(1)}\right)}{4 \lambda^{2}}<\left(u_{1}+x_{1}^{*}\right)\left|u_{2}+x_{2}^{*}\right| \leqslant \frac{\lambda^{2} d\left(\Lambda_{0}^{(1)}\right)}{4}
$$

and therefore

$$
-\frac{d\left(\Lambda_{0}\right)}{9 \lambda^{2}}<\left(u_{1}+x_{1}^{*}\right)\left|u_{2}+x_{2}^{*}\right|\left(n x_{33}+\bar{x}_{3}\right) \leqslant \frac{\lambda^{2} d\left(\Lambda_{0}\right)}{8} .
$$

The proof is then completed as before. Suppose now that $n x_{33}+\bar{x}_{3}<0$. By Lemma 2, there exists $\left(u_{1}, u_{2}\right) \in \Lambda_{0}^{(1)}$ such that

$$
-\frac{\lambda^{2} d\left(\Lambda_{0}^{(1)}\right)}{4}<\left(u_{1}+x_{1}^{*}\right)\left|u_{2}+x_{2}^{*}\right| \leqslant \frac{d\left(\Lambda_{0}^{(1)}\right)}{4 \lambda^{2}},
$$

and therefore also

$$
-\frac{d\left(\Lambda_{0}\right)}{8}<\left(u_{1}+x_{1}^{*}\right)\left|u_{2}+x_{2}^{*}\right|\left(n x_{33}+\bar{x}_{3}\right)<\frac{\lambda^{2} d\left(\Lambda_{0}\right)}{8} .
$$

The proof is then completed as before.

Case 5. $\left(x_{1}, x_{2}, x_{3}\right) \rightarrow\left(x_{3}, x_{2}, x_{1}\right)$ and $p=1$. By Lemma 1 , there exists $\left(u_{2}, u_{3}\right)$ $\in \Lambda_{0}^{(2)}$ such that

$$
-\frac{d\left(\Lambda_{0}^{(2)}\right)}{4 \lambda^{2}}<\left(u_{2}+\bar{x}_{2}\right)\left(u_{3}+\bar{x}_{3}\right) \leqslant \frac{\lambda^{2} d\left(\Lambda_{0}^{(2)}\right)}{4} .
$$

There exist integers $m, n$ such that

$$
\left(u_{2}, u_{3}\right)=m\left(x_{22}, x_{23}\right)+n\left(x_{32}, x_{33}\right),
$$


so put $x_{1}^{*}=m x_{21}+n x_{31}+\bar{x}_{1}$. Then, there exists $\left(u_{1}\right) \in \Lambda_{0}^{(1)}$ such that

$$
\left|u_{1}+x_{1}^{*}\right|<\frac{1}{2} d\left(\Lambda_{0}^{(1)}\right)
$$

and the proof is completed as before.

Case 6. $\left(x_{1}, x_{2}, x_{3}\right) \rightarrow\left(x_{3}, x_{2}, x_{1}\right)$ and $p=2$. The proof parallels that used in Case 4.

This completes the proof of Theorem 1A, and therefore of (a).

Proof OF (b).

LEMMA 8. If $0<b<2, \frac{1}{4} \leqslant s<4, t_{0}, x$ and $y$ are real numbers such that $-2 / b s \leqslant x y \leqslant 2 s / b$, and either $|x+y| \leqslant 3 / b$ or $|x-y| \leqslant 3 / b$, then there exists $t \equiv t_{0}$, modulo 1 , such that

$$
-1 / b s<(x+t)(y+t)|t|<s / b .
$$

Proof. First, choose $t \equiv t_{0}$, modulo 1 , so that $0<t<1$. We may assume that $t \neq 0$, otherwise the lemma is trivially true. We further assume that $x$ and $y$ do not satisfy the conclusion of the lemma and show that they do not satisfy the hypothesis. Thus, $x$ and $y$ do not satisfy either of the inequalities

$$
\begin{aligned}
-1 / b s t & <(x+t)(y+t)<s / b t \quad \text { and } \\
-1 / b s(1-t) & <(x+t-1)(y+t-1)<s / b(1-t) .
\end{aligned}
$$

Case A.

$$
\begin{gathered}
(x+t)(y+t)<-1 / b s t \quad \text { and } \\
(x+t-1)(y+t-1) \leqslant-1 / b s(1-t) .
\end{gathered}
$$

Multiplying (i) by $(1-t)$ and (ii) by $t$, and adding, we obtain

$$
x y+t^{2}(1-t)+t(t-1)^{2} \leqslant-\left(\frac{1-t}{t}+\frac{t}{1-t}\right) / b s<-2 / b s .
$$

Hence $x y<-2 / b s$, contrary to the hypothesis.

Case B.

$$
\begin{gathered}
(x+t)(y+t) \geqslant s / b t \quad \text { and } \\
(x+t-1)(y+t-1) \geqslant s / b(1-t) .
\end{gathered}
$$

Putting $t=\frac{1}{2}+e$, so that $|e|<\frac{1}{2}$, (i) and (ii) become

$$
\begin{gathered}
\left(x+e+\frac{1}{2}\right)\left(y+e+\frac{1}{2}\right)\left(\frac{1}{2}+e\right)>s / b \quad \text { and } \\
\left(x+e-\frac{1}{2}\right)\left(y+e-\frac{1}{2}\right)\left(\frac{1}{2}-e\right) \geqslant s / b .
\end{gathered}
$$


Multiplying these two inequalities together, we obtain

$$
\left((x+e)^{2}-\frac{1}{4}\right)\left((y+e)^{2}-\frac{1}{4}\right)\left(\frac{1}{4}-e^{2}\right)>s^{2} / b^{2} .
$$

Suppose first that $x+e+\frac{1}{2}>0$. This implies, by (i'), that $y+e+\frac{1}{2}>0$. If, further, $x+e-\frac{1}{2}<0$, then by (ii'), $y+e-\frac{1}{2}<0$, which implies that $|x+e|$ $<\frac{1}{2}$ and $|y+e|<\frac{1}{2}$. It follows from (iii) that

$$
\frac{1}{4} \cdot \frac{1}{4} \cdot \frac{1}{4} \geqslant s^{2} / b^{2}
$$

contrary to the inequalities $s \geqslant \frac{1}{4}$ and $b<2$. Hence, $x+e-\frac{1}{2}>0$, and therefore also by (ii'), $y+e-\frac{1}{2}>0$. Inequality (iii) then implies that

$$
(x+e)^{2}(y+e)^{2}\left(\frac{1}{4}-e^{2}\right)>s^{2} / b^{2} .
$$

If $e<0$, since $x>\frac{1}{2}-e$ and $y>\frac{1}{2}-e$, this inequality implies that

$$
x^{2} y^{2}>4 s^{2} / b^{2}
$$

contrary to the hypothesis. Therefore $e>0$. But then, by (ii'),

$$
x y>2 s / b \text {, }
$$

contrary to the hypothesis. This shows that we must have $x+e+\frac{1}{2}<0$.

It follows that $x+e-\frac{1}{2}<0$. Replacing $x, y$ and $e$ by $-x,-y$ and $-e$ respectively, interchanges inequalities (i') and (ii') while replacing the inequality $x+e-\frac{1}{2}<0$ by $x+e+\frac{1}{2}>0$. Hence we are lead to a contradiction exactly as before. This completes Case B.

Case $\mathrm{C}$.

$$
\begin{gathered}
(x+t)(y+t) \leqslant-1 / b s t \quad \text { and } \\
(x+t-1)(y+t-1)>s / b(1-t) .
\end{gathered}
$$

Setting $\zeta=x y$ and $\eta=x+y$ and subtracting (ii) from (i), we obtain

$$
\begin{aligned}
\eta & \leqslant-1 / b s t-s b(1-t)+1-2 t \\
& \leqslant-(2 / b \sqrt{t(1-t)})+1-2 t .
\end{aligned}
$$

If $\eta \geqslant-3 / b$ then this implies that

$$
-3 / b \leqslant-(2 / b \sqrt{t(1-t)})+1-2 t .
$$

As $b<2$, this implies that $t<\frac{1}{2}$ and

$$
\frac{2}{\sqrt{t(1-t)}}-3 \leqslant 2-4 t \text {. }
$$

Writing $t=\frac{1}{2}-e$, where $0<e<\frac{1}{2}$, this inequality may be written as

$$
0 \geqslant(7-10 e)(1-2 e)+96 e^{3}+64 e^{4},
$$


which is impossible, since all terms on the right side are positive. Therefore, to avoid a contradiction, it is necessary that $\eta<-3 / b$, so that $|x+y|>3 / b$.

By (i),

$$
\zeta \leqslant-t \eta-t^{2}-1 / b s t
$$

Hence

$$
(x-y)^{2}=\eta^{2}-4 \zeta \geqslant \eta^{2}+4 t \eta+4 t^{2}+4 / b s t .
$$

If $|x-y| \leqslant 3 / b$, this implies that

$$
9 / b^{2} \geqslant \eta^{2}+4 t \eta+4 t^{2}+4 / b s t .
$$

But $\eta^{2}+4 t \eta$ has a minimum at $\eta=-2 t$ and, by (iii),

$$
\eta \leqslant-(1 / b s t)-(s / b(1-t))+1-2 t \leqslant-2 t \text {. }
$$

Therefore, substituting this value for $\eta$ in (vi), we obtain

$$
\frac{9}{b^{2}} \geqslant \frac{1}{b s t}\left(\frac{1}{b s t}+2\right)+\left(\frac{s}{(1-t) b}-1\right)^{2}+\frac{2}{t(1-t) b^{2}} \text {. }
$$

Hence

$$
\frac{1}{b^{2}} \geqslant \frac{1}{b s t}\left(\frac{1}{b s t}+2\right)+\left(\frac{s}{(1-t) b}-1\right)^{2} .
$$

The first term on the right of (vii) is larger than $1 / b^{2}$ if $s t<1$, hence $s t>1$. But then $s t>4 t(1-t)$, that is $s /(1-t)>4$. As $b<2$, (vii) then implies that

$$
\frac{1}{b}>\frac{4}{b}-1 \quad \text { or } \quad b>3 \text {, }
$$

contrary to hypothesis. It follows that $|x-y|>3 / b$.

Case D.

$$
\begin{gathered}
(x+t)(y+t) \geqslant s / b t \\
(x+t-1)(y+t-1) \leqslant-1 / b s(1-t) .
\end{gathered}
$$

Changing $x, y$ into $-x,-y$, and replacing $t$ by $1-t$, turns inequalities (i) and (ii) into the corresponding inequalities for Case $C$, without changing $|\eta|$. Hence, by symmetry, Case $\mathrm{D}$ follows from Case $\mathrm{C}$.

This completes the proof of Lemma 8.

Lemma 9. If $\left(x_{1}, x_{2}, x_{3}\right) \in R_{3}$ and $\Lambda$ is a lattice in $R_{3}$, then there exists a point $\left(z_{1}, z_{2}, z_{3}\right) \in \Lambda$ such that

$$
\begin{aligned}
& z_{1}+x_{1}>0, \quad z_{2}+x_{2}>0 \quad \text { and } \\
& \left(z_{1}+x_{1}\right)\left(z_{2}+x_{2}\right)\left|z_{3}+x_{3}\right| \leqslant \frac{1}{2} d(\Lambda),
\end{aligned}
$$

with equality only if $m_{h}(\Lambda)=0$. 
This lemma is due to Cole [4].

Corollary. If $\lambda<\frac{1}{2}$ or $\lambda>2$, then Theorem 1 is true.

Proof. If $\lambda>2$, the solution given by Lemma 9 clearly satisfies Theorem 1 . If $\lambda<\frac{1}{2}$, Lemma 9 asserts that there exists a point $\left(z_{1}, z_{2}, z_{3}\right) \in \Lambda$ such that $z_{1}+x_{1}>0, z_{2}+x_{2}<0$, and

$$
\left(z_{1}+x_{1}\right)\left(-z_{2}-x_{2}\right)\left|z_{3}+x_{3}\right| \leqslant \frac{1}{2} d(\Lambda) .
$$

Here we have applied Lemma 9 to the point $\left(x_{1},-x_{2}, x_{3}\right)$ and the lattice obtained from $\Lambda$ by changing the signs of the second coordinates. Hence

$$
-\frac{1}{2} d(\Lambda) \leqslant\left(z_{1}+x_{1}\right)\left(z_{2}+x_{1}\right)\left(z_{2}+x_{2}\right)\left|z_{3}+x_{3}\right| \leqslant 0,
$$

giving a solution satisfying Theorem 1 , unless the equality sign holds on the left, in which case $m_{h}(\Lambda)=0$. Since this has been taken care of by Theorem $1 \mathrm{~A}$, the corollary follows.

THEOREM 1B. If $m_{h}(\Lambda)>0$ and is an attained minimum then Theorem 1 is true.

Proof. We assume, by way of contradiction, that Theorem 1 is false for $\Lambda$ with some point $X$ and a value of $\lambda^{2}=s$, say. Then strict inequality in Theorem 1 cannot be attained for any point of $\Lambda$. By homogeneity, we may further assume that $d(\Lambda)=8$. The corollary to Lemma 9 implies that $\frac{1}{4}<s<4$. Set $m_{h}(\Lambda)=b$, and let $a$ be a positive number for which $a^{3}=b$. As Theorem 1 is invariant under standard transformations and $m_{h}(\Lambda)$ is attained, we may assume that the point $( \pm a, \pm a, \pm a) \in \Lambda$ for at least one choice of signs. Further, changing the sign of a coordinate leaves the inequality of Theorem 1 invariant, apart from possibly replacing $s$ by $s^{-1}$. Hence, we may assume that $(a, a, a) \in \Lambda$. By a well-known theorem of Davenport [7], it follows that $d(\Lambda)>7 b$, hence $b \leqslant 8 / 7$.

Denote by $\Lambda_{2}$, the two-dimensional lattice obtained by projecting $\Lambda$ into the $x, y$ plane, parallel to the vector $(a, a, a)$. Then the projection of $a^{-1} \Lambda$ is $a^{-1} \Lambda_{2}$. As $m_{h}\left(a^{-1} \Lambda\right)=1$, applying Lemma 8 with $b=s=1$, it follows that $a^{-1} \Lambda_{2}$ has no point $(x, y) \neq(0,0)$ such that

$$
-2 \leqslant x y \leqslant 2,
$$

and either $|x+y| \leqslant 3$ or $|x-y| \leqslant 3$. Hence $a^{-1} \Lambda_{2}$ has no such point for which

$$
|x|+|y| \leqslant 2 \sqrt{2} \text {, }
$$

and therefore $\Lambda_{2}$ has no such point for which

$$
|x|+|y| \leqslant 2 \sqrt{2} a \text {. }
$$


Let $(\xi, \eta) \neq(0,0)$, be a point of $\Lambda_{2}$ such that $\Lambda_{2}$ has no point $(x, y) \neq(0,0)$ for which

$$
|x|+|y|<|\xi|+|\eta|
$$

By Minkowski's fundamental theorem,

$$
|\xi|+|\eta| \leqslant 4 a^{-1 / 2}, \text { since } d\left(\Lambda_{2}\right)=8 a^{-1} .
$$

Because of the symmetry in Lemma 8 , there is no loss of generality in assuming that $\eta \geqslant \xi \geqslant 0$.

In the notation of Theorem 1 , if $\bar{X}$ denotes the projection of $X$ into the $x, y$ plane parallel to the vector $(a, a, a)$, then $\Lambda_{2}+\bar{X}$ is the image of $\Lambda+X$ under this projection. Applying Lemma 8 with $b=a^{3}$ and $s=\lambda^{2}$, we claim that $\Lambda_{2}+\bar{X}$ has no point in the region $K$ in the plane given as the set of points $(x, y)$ such that

$$
-\frac{2}{a s} \leqslant x y \leqslant \frac{2 s}{a},
$$

and either $|x+y| \leqslant 3 / a^{2}$ or $|x-y| \leqslant 3 / a^{2}$. To see this, assume by way of contradiction that $\Lambda_{2}+\bar{X}$ contains such a point $(x, y)$. Then $x / a, y / a$ satisfy the hypothesis of Lemma 8 . Therefore, given any real number $t_{0}$, there exists $t \equiv t_{0}(\bmod 1)$ such that

$$
-\frac{1}{s a^{3}}<\left(\frac{x}{a}+t\right)\left(\frac{y}{a}+t\right)|t|<\frac{s}{a^{3}} .
$$

Hence, there exists $t^{\prime} \equiv a t_{0}(\bmod a)$ such that

$$
-\lambda^{-2}<\left(x+t^{\prime}\right)\left(y+t^{\prime}\right)\left|t^{\prime}\right|<\lambda^{2} .
$$

As $(x, y) \in \Lambda_{2}+\bar{X}$ and $(a, a, a) \in \Lambda$, there exists a real number $c$ such that $(x+a c, y+a c, a c) \in \Lambda+X$. Taking $t_{0}=c$ in the preceding argument, we find that $\left(x+t^{\prime}, y+t^{\prime}, t^{\prime}\right) \in \Lambda+X$, and Theorem 1 is true for $\Lambda, X$ and $\lambda^{2}$ with strict inequality, contrary to the hypothesis. This establishes the claim.

We obtain the desired contradiction proving Theorem 1 by showing that $\Lambda_{2}+\bar{X}$ does contain a point of $K$.

Assume first that $\Lambda_{2}+\bar{X}$ contains no point $(x, y)$ such tat $0 \leqslant x \leqslant \xi$ and $0<y<\eta$. The projection of $\Lambda_{2}+\bar{X}$ into the $x$-axis, parallel to $(\xi, \eta)$, is a grid of determinant $8(a \eta)^{-1}$. Let $(\gamma, 0)$ be a point of this projection that is closest to 0 . Then

$$
|\gamma| \leqslant 4(a \eta)^{-1}
$$

In what follows, there is no loss of generality in assuming $\gamma>0$. As $\eta \geqslant \xi$ and $|\xi|+|\eta| \geqslant 2 \sqrt{2} a$, it follows that $\eta \geqslant \sqrt{2} a$, and so

$$
\gamma \leqslant 2 \sqrt{2} a^{-2} \text {. }
$$


For some real $t$ with $0<t<1$, we have, say,

$$
\begin{gathered}
\left(x_{1}, y_{1}\right)=(\gamma, 0)+t(\xi, \eta)=P_{1} \in \Lambda_{2}+\bar{X} \quad \text { and } \\
\left(x_{2},-y_{2}\right)=(\gamma, 0)+(t-1)(\xi, \eta)=P_{2} \in \Lambda_{2}+\bar{X},
\end{gathered}
$$

where $x_{1}, x_{2}, y_{1}, y_{2}$ are non-negative real numbers.

We assert that neither $P_{1}$ nor $P_{2}$ lies in the region given by

$$
\left\{(x, y)|| x+y \mid>3 a^{-2}\right\} \cap\left\{(x, y)|| x-y \mid>3 a^{-2}\right\} \text {. }
$$

To prove this assertion, assume first that $\left|x_{1}-y_{1}\right|>3 a^{-2}$. As

$$
x_{1}-y_{1}=\gamma+t(\xi-\eta)<\gamma \leqslant 2 \sqrt{2} a^{-2}<3 a^{-2},
$$

so

$$
y_{1}-x_{1}>3 a^{-2} \text {. }
$$

Now $x_{2}+y_{2} \geqslant 0$ and $x_{2}-y_{2}<\gamma<3 a^{-2}$. Hence, if $x_{2}-y_{2}<-3 a^{-2}$ and $x_{2}+y_{2}>3 a^{-2}$ then $y_{2}>3 a^{-2}$, that is $(1-t) \eta>3 a^{-2}$. But (i) implies that t $\eta>3 a^{-2}$. Hence $\eta>6 a^{-2}$. As $\xi+\eta<4 a^{-1 / 2}$, it follows that $\eta<4 a^{-1 / 2}$. Whence $a^{3}>9 / 4$, which is a contradiction. Therefore, either $\left|x_{2}+y_{2}\right|<3 a^{-2}$ or $\left|x_{2}-y_{2}\right| \leqslant 3 a^{-2}$. If $P_{2}=\left(x_{2}, y_{2}\right) \in K$, we have a contradiction, hence $x_{2} y_{2}$ $>2 /$ as $\geqslant \frac{1}{2} a^{-1}$, since $s \leqslant 4$; that is

$$
(\gamma+(t-1) \xi)(t-1) \eta<-\frac{1}{2} a^{-1} .
$$

Inequalities (i) and (ii) imply that

$$
t(\eta-\xi)-3 a^{-2}>\gamma>(1-t) \xi+(2 a(1-t) \eta)^{-1} .
$$

Hence

$$
t \eta-(2 a(1-t) \eta)^{-1}>3 a^{-2} .
$$

The left hand side of this inequality is negative at $t=0$, and tends to $-\infty$ as $t$ tends to 1 from below. Hence it must have a maximum in $0<t<1$, for the inequality to hold. This maximum is then achieved at $t=1-1 / \eta \sqrt{2 a}$ and $t$ may be replaced in the inequality by this value to give

$$
\eta>3 a^{-2}+\sqrt{2} a^{-1 / 2} \text {. }
$$

But as $\eta \leqslant 4 a^{-1 / 2}$, this contradicts $a^{3}<8 / 7$. Therefore we must have $\left|x_{1}-y_{1}\right|$ $\leqslant 3 a^{-2}$.

Now assume that $P_{2}$ satisfies $\left|x_{2}+y_{2}\right|>3 a^{-2}$. As

$$
x_{2}-y_{2}=\gamma+(t-1)(\xi+\eta)<3 a^{-2},
$$

it follows that

$$
x_{2}-y_{2}<-3 a^{-2}
$$


Since $P_{1}=\left(x_{1}, y_{1}\right)$ does not lie in $K$ and $\left|x_{1}-y_{1}\right|<3 a^{-2}$, it follows that $x_{1} y_{1}>2 s / a$, that is

$$
(\gamma+t \xi) t \eta>\frac{2 s}{a} \geqslant \frac{1}{2} a^{-1}, \quad \text { since } s \geqslant \frac{1}{4} .
$$

Inequalities (i') and (ii') imply that

$$
(2 a t \eta)^{-1}-t \xi<\gamma<(1-t)(\xi+\eta)-3 a^{-2},
$$

and therefore also

$$
\eta \eta(2 a t \eta)^{-1}<4 a^{-1 / 2}-3 a^{-2} .
$$

For $0 \leqslant t$, the left hand side has a minimum at $t=1 / \eta \sqrt{2 a}$, so $\sqrt{2} a^{-1 / 2}<$ $4 a^{-1 / 2}-3 a^{-2}$, again contrary to $a^{3}<8 / 7$. Therefore $P_{2}$ lies in $|x+y|<3 a^{-2}$. This proves our assertion concerning $\boldsymbol{P}_{1}$ and $\boldsymbol{P}_{2}$.

As $P_{1}$ and $P_{2}$ do not lie in $K$, it follows that $x_{1} y_{1}>2 a^{-1} s$ and $x_{2} y_{2}>2(a s)^{-1}$. Hence

$$
\begin{aligned}
x_{1} y_{2}+x_{2} y_{1} & =\left(x_{1} y_{1}\right) y_{2} y_{1}^{-1}+\left(x_{2} y_{2}\right) y_{1} y_{2}^{-1} \\
& >2\left(s y_{2} y_{1}^{-1}+y_{1}\left(s y_{2}\right)^{-1}\right) a^{-1}<4 a^{-1} .
\end{aligned}
$$

However, from the choice of $\gamma$, it follows that

$$
d\left(\Lambda_{2}\right)>8 a^{-1},
$$

which is a contradiction.

We have therefore shown that $\Lambda_{2}+\bar{X}$ contains a point $\left(x_{0}, y_{0}\right)$ such that $0<x_{0}<\xi$, and $0 \leqslant y_{0} \leqslant \eta$.

As $\left(x_{0}, y_{0}\right)-(\xi, \eta)$ cannot lie in $K$, either

$$
x_{0}-\xi+y_{0}-\eta<-3 a^{-2},
$$

in which case $x_{0}+y_{0}<4 a^{-1 / 2}-3 a^{-2}$; or

$$
\left(x_{0}-\xi\right)\left(y_{0}-\eta\right)>2 a^{-1} s \geqslant \frac{1}{2} a^{-1},
$$

in which case, by the arithmetic-geometric mean inequality,

$$
\xi-x_{0}+\eta-y_{0}>\sqrt{2} a^{-1 / 2} \text {, }
$$

so that $x_{0}+y_{0}<(4-\sqrt{2}) a^{-1 / 2}$. As $\sqrt{2} a^{-1 / 2}<3 a^{-2}$, it follows that in either case,

$$
x_{0}+y_{0}<(4-\sqrt{2}) a^{-1 / 2}<3 a^{-2} .
$$

Therefore, as $\left(x_{0}, y_{0}\right)$ is not in $K$, it follows that

$$
x_{0} y_{0}>2 a^{-1} s \text {. }
$$


Similarly, it follows that

$$
\begin{gathered}
\xi-x_{0}+\eta-y_{0}<(4-\sqrt{2}) a^{-1 / 2}, \quad \text { and } \\
\left(\xi-x_{0}\right)\left(\eta-y_{0}\right)>2 a^{-1} s .
\end{gathered}
$$

Therefore, again by the arithmetic-geometric mean inequality,

$$
x_{0}+y_{0}>2 \sqrt{\frac{2 s}{a}} \quad \text { and } \quad \xi-x_{0}+\eta-y_{0}>2 \sqrt{\frac{2 s}{a}} .
$$

Adding these two inequalities, we obtain $\xi+\eta>4 \sqrt{2 s / a}$. As $\xi+\eta<4 a^{-1 / 2}$, this implies that $s<\frac{1}{2}$.

Now redefine the point $\left(x_{1}, y_{1}\right)$ as that point of $\Lambda_{2}+\bar{X}$ for which $x_{1}>0$, $y_{1} \geqslant 0$ and $x_{1}+y_{1}=\alpha$ is as small as possible. Similarly, redefine the point $\left(x_{2}, y_{2}\right)$ as that point of $\Lambda_{2}+\bar{X}$ for which $x_{2}<0, y_{2}<0$ and $x_{2}+y_{2}=-\beta$ is as large as possible. By what has already been shown,

$$
\alpha, \beta \leqslant(4-\sqrt{2}) a^{-1 / 2}<3 a^{-2},
$$

hence $x_{1} y_{1}>2 a^{-1} s$ and $x_{2} y_{2}>2 a^{-1} s$. Let $R$ denote the rectangle of points $(x, y)$ given by

$$
-\beta \leqslant x+y \leqslant \alpha \quad \text { and } \quad|x-y| \leqslant 4 a^{-1 / 2} .
$$

As $s<\frac{1}{2}$, so $R$ is contained in the set of points $(x, y)$ given by

$$
x y \geq-2(s a)^{-1} \text { and } \min (|x+y|,|x-y|)<3 a^{-2} \text {. }
$$

Therefore, $R$ contains no point of $\Lambda_{2}+\bar{X}$ in its interior. It is easy to see that each vertex of $R$ has coordinates of opposite sign. Hence, $R$ contains the points $\left(x_{1}, y_{1}\right),\left(x_{2}, y_{2}\right)$ on its boundary. Now, all points of $\Lambda_{2}+\bar{X}$ lie on lines parallel to the segment $\left(x_{1}, y_{1}\right)-\left(x_{2}, y_{2}\right)$ and spaced so that any two adjacent points on one such line are the endpoints of a segment congruent to $\left(x_{1}, y_{1}\right)-\left(x_{2}, y_{2}\right)$. It follows that there exist numbers $\rho, \sigma \geqslant 4 a^{-1 / 2}$, such that the rectangle $R^{\prime}$ of points $(x, y)$ given by

$$
-\beta \leqslant x+y \leqslant \alpha \quad \text { and } \quad-\rho \leqslant x-y<\sigma,
$$

contains points $\left(x_{3}, y_{3}\right)$ and $\left(x_{4}, y_{4}\right)$ of $\Lambda_{2}+\bar{X}$ such that

$$
x_{3}-y_{3}=-\rho \quad \text { and } \quad x_{4}-y_{4}=\sigma \text {; }
$$

and such that $R^{\prime}$ contains no point of $\Lambda_{2}+\bar{X}$ in its interior. Therefore $x_{3} y_{3}<-2(a s)^{-1}$ and $x_{4} y_{4}<-2(a s)^{-1}$. If neither point is a vertex of $R^{\prime}$, then the two triangles with vertices $\left(x_{1}, y_{1}\right),\left(x_{2}, y_{2}\right),\left(x_{3}, y_{3}\right)$ and $\left(x_{1}, y_{1}\right),\left(x_{2}, y_{2}\right),\left(x_{4}, y_{4}\right)$ contain no point of $\Lambda_{2}+\bar{X}$ apart from their vertices. It follows that the four points form the vertices of a divided cell of $\Lambda_{2}+\bar{X}$, which therefore has area $8 a^{-1}$, contradicting Lemma 5. Hence, at least one of $\left(x_{3}, y_{3}\right),\left(x_{4}, y_{4}\right)$ is a vertex of $R^{\prime}$. This implies that the lines $x+y=-\beta$ and $x+y=\alpha$ are two adjacent lines of lattice points of $\Lambda_{2}+\bar{X}$. Neither of these lines can intersect a coordinate 
axis in a point of $\Lambda_{2}+\bar{X}$ because such a point $(x, y)$ satisfies $|x+y|<3 a^{-2}$ and therefore $|x y|>\min \left(2 a^{-1} s, 2 a^{-1} s^{-1}\right)$. It follows that there exist four points $\left(x_{i}^{\prime}, y_{i}^{\prime}\right), i=1,2,3,4$ forming the vertices of a divided cell of $\Lambda_{2}+\bar{X}$, and satisfying

$$
\begin{array}{ll}
x_{i}^{\prime} y_{i}^{\prime}>2 a^{-1} s & \text { for } i=1,2 ; \\
x_{i}^{\prime} y_{i}^{\prime}<-2(a s)^{-1} & \text { for } i=3,4 ;
\end{array}
$$

again contradicting Lemma 5 . This proves the theorem.

\section{References}

[1] B. J. Birch \& H. P. F. Swinnerton-Dyer, 'On the inhomogeneous minimum of the product of $n$ linear forms' Mathematika 3 (1956), 25-39.

[2] H. Blaney, 'Some asymmetric inequalities' Proc. Cambridge Philos. Soc. 46 (1950), 359-376.

[3] J. W. S. Cassels, An introduction to the geometry of numbers, p. 326 (Springer-Verlag, (1959)).

[4] A. J. Cole, 'On the product of $n$ linear forms' Quart. J. Math. Oxford Ser. 3 (1952), 56-62.

[5] H. Davenport, 'A simple proof of Remak's theorem on the product of three linear forms' $J$. London Math. Soc. 14 (1939), 47-51.

[6] H. Davenport, 'Non-homogeneous ternary quadratic forms' Acta Math. 80 (1948), 65-95.

[7] H. Davenport, 'On the product of three homogeneous linear forms' Proc. London Math. Soc. (2) 44 (1938), 412-431. See also

H. Davenport, 'Note on the product of three homogeneous linear forms' $J$. London Math. Soc. 16 (1941), 98-101.

[8] K. Mahler, 'On a property of positive definite ternary quadratic forms' J. London Math. Soc. 15 (1940), 305-320.

[9] K. Mahler, 'On lattice points in $n$-dimensional star bodies. I Existence theorems' Proc. Roy. Soc. London Ser. A 187 (1946), 151-187.

[10] H. Minkowski, Gesammelte Abhandlungen I, p. 342 (Leipzig, Berlin (1911)).

[11] KH. N. Narzullaev, The representation of a unimodular matrix in the form DOTU for $n=3$ ' Math. Notes 18 (1975), 713-719.

[12] R. Remak, 'Verellgemeinerung eines Minkowskischen Satzes' I, II Math. Z. 17 (1923), 1-34; 18 (1923), 173-200.

[13] D. B. Sawyer, 'The product of two non-homogeneous linear forms' J. London Math. Soc. 23 (1948), 250-251.

[14] A. C. Woods, 'Divided cells and a conjecture of Minkowski', to appear.

\section{Department of Mathematics}

The Ohio State University

Columbus, Ohio 43210

U.S.A. 